\title{
Document Version Revision Reason
}

National Cancer Institute

\section{Source}

National Cancer Institute. Document Version Revision Reason. NCI Thesaurus. Code C93819.

The reason why the document is revised. 